J. Phys. IV France 130 (2005) 115-132

(C) EDP Sciences, Les Ulis

DOI: $10.1051 /$ jp4:2005130008

\title{
Diffusion incohérente des neutrons : modèles analytiques pour la dynamique interne des protéines
}

\author{
D.J. Bicout ${ }^{1}$ \\ 1 Institut Laue - Langevin, Theory Group, 6 rue Jules Horowitz, BP. 156, \\ 38042 Grenoble Cedex 9, France \\ et \\ Unité Biomathématiques et Epidémiologie - ENVL, 1 Av. Bourgelat, BP. 83, \\ 69280 Marcy l'Étoile, France
}

\begin{abstract}
Résumé. La dynamique interne des protéines joue un rôle central dans la stabilité, la fonction et l'activité biologique de ces biomolécules. Il est maintenant établi que les fluctuations d'états conformationnels des protéines influencent fortement la plupart des réactions biochimiques et s'accompagnent d'une augmentation brutale des déplacements carrés moyens des atomes au dessus de la température de la transition dynamique. Dans cette contribution, nous présentons une revue critique de quelques modèles théoriques couramment utilisés dans la littérature pour l'analyse des mouvements internes des protéines et la description de la transition dynamique.
\end{abstract}

Table des matières

1 INTRODUCTION

2 FONCTIONS DE LA DIFFUSION INCOHÉRENTE DES NEUTRONS

3 DYNAMIQUE INTERNE DES PROTÉINES : MODÈLES CLASSIQUES

3.1 Saut ou Transition entre deux Sites non - Équivalents . . . . . . . . . . . . . . . . 119

3.2 Diffusion à l'intérieur d'une Sphère . . . . . . . . . . . . . . . . . . 120

4 EXTENSIONS DES MODÈLES CLASSIQUES : HÉTÉROGÉNÉITÉ DYNAMIQUE 122

4.1 Dynamiques Additive et Multiplicative [32] . . . . . . . . . . . . . . . . 122

4.1 .1 Dynamique Additive . . . . . . . . . . . . . . . . . . . . . . . . . 122

4.1 .2 Dynamique Multiplicative . . . . . . . . . . . . . . . . . 123

4.1.3 Comportements limites des Dynamiques Additive et Multiplicative . . . . . . 124

4.2 Modèle en Couche des Dynamiques Additive et Multiplicative . . . . . . . . . . . 125

5 MODÈLE DE DIFFUSION À L'INTÉRIEUR DE DEUX SPHÈRES CONCENTRIQUES

6 TRANSITION DYNAMIQUE : CONSTANTES DE FORCE INTERNE D'UNE PROTÉINE

7 CONCLUSION ET PERSPECTIVE

\section{INTRODUCTION}

Les biomolécules sont des systèmes dynamiques dont la stabilité, la fonction et l'activité biologique sont régulées de manière cruciale par leur dynamique interne. Cette dynamique interne inclue une variété 
de mouvements et changements conformationnels qui couvrent plusieurs ordre de grandeurs dans les amplitudes $(0.01-100 \AA)$ et les échelles de temps $\left(10^{-15}-1 \mathrm{~s}\right)[1,2]$. Bien que la relation entre la dynamique et la fonction des biomolécules ne soit pas encore élucidée, il est clairement établi que pour fonctionner les biomolécules doivent expérimenter des changements d'états conformationnels [3, 4]. Ces mouvements et transitions conformationnels des biomolécules influencent fortement et jouent un rôle important dans la plupart des réactions biochimiques comme la catalyse des enzymes, la liaison de récepteur-ligand, et dans d'autres aspects de l'activité biologique tels le repliement des protéines, le transfert de charges au travers des membranes protéiques, etc. Cependant, on observe expérimentalement que l'activité (cinétique des réactions biochimiques) des biomolécules hydratées accuse une baisse notable en dessous de la température de $\mathrm{T} \sim 250-200 \mathrm{~K}$ [5-7]. Par ailleurs, plusieurs expériences ont mis en évidence l'existence d'une transition dynamique dans les systèmes protéiques hydratés, c'est à dire que les déplacements carrés moyens des atomes dans les protéines hydratées augmentent brutalement au dessus de la température T $180-200 \mathrm{~K}$ [6-18]. Cette coincidence dans le changement de comportement des systèmes protéiques hydratés en fonction de la température amène à considérer que la transition dynamique et l'activité des biomolécules pourraient être des événements liés régis par les mêmes processus ou mécanismes.

Dans cette présentation nous nous intéressons aux mouvements moléculaires thermiques associés aux forces qui maintiennent la structure tertiaire et quaternaire des protéines. Ces forces ont pour origine le reseau des liaisons hydrogènes, les interactions électrostatiques et de Van der Waals, et les forces associées à l'effet hydrophobe [19]. A cause de leur longueur d'onde (1 Å) et énergie (1 kcal/mol), les techniques de diffusion des neutrons permettent d'étudier ces mouvements thermiques des biomolécules qui sont associés á des énergies de l'ordre de quelques $\mathrm{kcal} / \mathrm{mol}$, des fluctuations atomiques de quelques angström, et des échelles de temps allant de la pico á la nanoseconde. Considérant que la réalité de la dynamique intramoléculaire des protéines peut être accessible par la théorie et l'expérience, notre objectif dans ces notes est de discuter des modèles qui permettent (1) - de reconsidérer notre manière d'analyser les données expérimentales et l'interprétation des paramètres déterminés, et (2) d'améliorer notre compréhension des phénomènes étudiés et d'identifier les paramètres importants du probléme.

Pour ce faire, nous présentons une revue de quelques modèles théoriques couramment utilisés dans la littérature pour l'analyse des mouvements internes des protéines. Le choix de ces modèles est basé sur leur caractère simple et pédagogique, sur la possibilité de dériver des résultats analytiques des fonctions intéressantes, et sur le fait que les déplacements carrés moyens associés présentent le comportement de la transition dynamique. Plus précisément, nous traiterons des deux modèles classiques de diffusion dans une sphére imperméable et de dynamique de sauts entre deux sites non équivalents. Des extensions de ces modèles aux modèles de dynamiques combinées seront abordées dans le cadre de l'hétérogénéité dynamique. Nous discuterons ensuite du modèle de diffusion dans deux sphére concentriques comme synthése des deux modèles classiques. Enfin, nous terminerons cette présentation par une description simple de la transition dynamique.

\section{FONCTIONS DE LA DIFFUSION INCOHÉRENTE DES NEUTRONS}

Dans cette section, nous introduisons et donnons les définitions des fonctions qui seront utilisées pour la discussion sur les modèles de la dynamique interne des protéines [20-22]. Pour la sonde neutron, ce sont les atomes d'hydrogènes, attachés à d'autres groupements chimiques de la structure protéique, qui sont les révélateurs des mouvements internes de la protéine. De ce fait, nous considérons la diffusion incohérente d'un faisceau de neutrons par les atomes d'hydrogénes associés d'une protéine. Ces atomes d'hydrogènes (que nous appelerons aussi particules) sont en mouvement dans un potentiel $V(\mathbf{r})$, où $\mathbf{r}(t)$ représente le vecteur position de l'ensemble des particules à l'instant $t$. La quantité intéressante mesurée dans les expériences de diffusion incohérente de neutrons est le facteur de forme ou de structure 
dynamique incohérent,

$$
S_{\mathrm{inc}}(\mathbf{Q}, \omega)=\int_{-\infty}^{\infty} d \omega^{\prime} R\left(\omega-\omega^{\prime}\right) \frac{1}{\pi} \int_{0}^{\infty} I(\mathbf{Q}, t) \cos \left(\omega^{\prime} t\right) d t,
$$

où $\hbar \omega$ est l'énergie échangée entre le faisceau de neutron et le système, et $R\left(\omega-\omega^{\prime}\right)$ (tel que $\int_{-\infty}^{\infty} d \omega R(\omega)=1$ ) est la fonction de résolution expérimentale que nous prendrons de la forme:

$$
R(\omega)=\left\{\begin{array}{l}
\tau / 2 ;-1 \leq \omega \tau \leq 1 \\
0 ; \quad \text { ailleurs }
\end{array}\right.
$$

où $\tau$ désigne le temps de résolution du système experimental utilisé. La fonction intermédiaire d'intensité diffusée incohérente $I(\mathbf{Q}, t)$ est définie par,

$$
\begin{aligned}
I(\mathbf{Q}, t) & =\sum_{\alpha=1}^{N} x_{\alpha}\left\langle\mathrm{e}^{i \mathbf{Q} \cdot \mathbf{r}_{\alpha}(t)} \mathrm{e}^{-i \mathbf{Q} \cdot \mathbf{r}_{\alpha}(0)}\right\rangle \\
& =\sum_{\alpha=1}^{N} x_{\alpha} \int d \mathbf{r}_{0} \int d \mathbf{r} \mathrm{e}^{i \mathbf{Q} \cdot \mathbf{r}_{\alpha}} G\left(\mathbf{r}, t \mid \mathbf{r}_{0}\right) \mathrm{e}^{-i \mathbf{Q} \cdot \mathbf{r}_{0 \alpha}} P_{\mathrm{eq}}\left(\mathbf{r}_{0}\right) \\
& =\sum_{\alpha=1}^{N} x_{\alpha} \int d \mathbf{r}_{0 \alpha} \int d \mathbf{r}_{\alpha} \mathrm{e}^{i \mathbf{Q} \cdot \mathbf{r}_{\alpha}} G_{\alpha}\left(\mathbf{r}_{\alpha}, t \mid \mathbf{r}_{0 \alpha}\right) \mathrm{e}^{-i \mathbf{Q} . \mathbf{r}_{0 \alpha}} P_{\alpha \mathrm{eq}}\left(\mathbf{r}_{0 \alpha}\right) .
\end{aligned}
$$

Dans cette expression, $x_{\alpha}$ (avec $\sum_{\alpha=1}^{N} x_{\alpha}=1$ ) représente la proportion de particules dynamiquement équivalents (i.e., effectuant les mêmes mouvements dans un potentiel identique $U_{\alpha}(\mathbf{r})$ ), $\mathbf{Q}$ est le vecteur d'onde diffusée des neutrons, et $\langle\cdots\rangle$ représente la moyenne d'ensemble sur plusieurs trajectoires de la population des particules initialement à l'équilibre thermique. La fonction de Green réduite (ou fonction self de Van Hove) $G_{\alpha}\left(\mathbf{r}_{\alpha}, t \mid \mathbf{r}_{0 \alpha}\right)$ est le propagateur décrivant la nature des mouvements des particules de type $\alpha$ dans le potentiel de force moyenne $U_{\alpha}(\mathbf{r})$, i.e. la densité de probabilité de trouver la particule à la position $\mathbf{r}_{\alpha}$ à l'instant $t$ sachant qu'elle était initialement en $\mathbf{r}_{0 \alpha}$. La distribution $P_{\alpha e q}\left(\mathbf{r}_{\alpha}\right)$ à l'équilibre thermique de ces particules de type $\alpha$ est donnée par la relation,

$$
P_{\alpha \mathrm{eq}}\left(\mathbf{r}_{\alpha}\right)=\frac{\mathrm{e}^{-\beta U_{\alpha}\left(\mathbf{r}_{\alpha}\right)}}{\int \mathrm{e}^{-\beta U_{\alpha}\left(\mathbf{r}_{\alpha}\right)} d \mathbf{r}_{\alpha}} \quad ; \quad \beta^{-1}=\mathrm{k}_{\mathrm{B}} \mathrm{T}, \text { energie thermique } .
$$

Il vient de ce qui précède que $I(\mathbf{Q}, t)=\sum_{\alpha=1}^{N} x_{\alpha} I_{\alpha}(\mathbf{Q}, t)$ et $S_{\text {inc }}(\mathbf{Q}, \omega)=\sum_{\alpha=1}^{N} x_{\alpha} S_{\alpha}(\mathbf{Q}, \omega)$, où $I_{\alpha}(\mathbf{Q}, t)$ et $S_{\alpha}(\mathbf{Q}, \omega)$ sont reliés par la transformée de Fourier comme dans Eq. (2.1).

Pour chaque particule de type $\alpha$, la fonction intermédiaire d'intensité diffusée incohérente peut s'écrire sous la forme,

$$
I_{\alpha}(\mathbf{Q}, t)=\left\{A_{0 \alpha}(\mathbf{Q})+\left[1-A_{0 \alpha}(\mathbf{Q})\right] C_{\alpha}(\mathbf{Q}, t)\right\} \times \text { Debye-Waller } \alpha(\mathbf{Q})
$$

où Debye-Waller $r_{\alpha}(\mathbf{Q})$ est le facteur de Debye-Waller (que nous allons omettre dans la suite par soucis de simplicité), $A_{0 \alpha}(\mathbf{Q})$ est le facteur de forme ou de structure incohérent élastique (EISF - Elastic Incoherent Structure Factor) défini par la relation,

$$
A_{0 \alpha}(\mathbf{Q})=\left|\left\langle\mathrm{e}^{i \mathbf{Q} \cdot \mathbf{r}_{\alpha}}\right\rangle\right|^{2}=\left|\int \mathrm{e}^{i \mathbf{Q} \cdot \mathbf{r}_{\alpha}} P_{\alpha \mathrm{eq}}\left(\mathbf{r}_{\alpha}\right) d \mathbf{r}_{\alpha}\right|^{2},
$$

et $C_{\alpha}(\mathbf{Q}, t)$ la fonction de corrélation incohérente (ICF - Incoherent scattering Correlation Function), donnée par la relation,

$$
C_{\alpha}(\mathbf{Q}, t)=\frac{\left\langle\mathrm{e}^{i \mathbf{Q} . \mathbf{r}_{\alpha}(t)} \mathrm{e}^{-i \mathbf{Q} \cdot \mathbf{r}_{\alpha}(0)}\right\rangle-\left|\left\langle\mathrm{e}^{i \mathbf{Q} \cdot \mathbf{r}_{\alpha}}\right\rangle\right|^{2}}{1-\left|\left\langle\mathrm{e}^{i \mathbf{Q} \cdot \mathbf{r}_{\alpha}}\right\rangle\right|^{2}}
$$


avec un temps de corrélation $1 / k_{\alpha}(Q)$ donné par,

$$
\frac{1}{k_{\alpha}(\mathbf{Q})}=\int_{0}^{\infty} C_{\alpha}(\mathbf{Q}, t) d t
$$

De même, le facteur de structure dynamique s'écrit,

$$
S_{\alpha}(\mathbf{Q}, \omega)=A_{0 \alpha}(\mathbf{Q}) R(\omega)+\left[1-A_{0 \alpha}(\mathbf{Q})\right] \int_{-\infty}^{\infty} d \omega^{\prime} R\left(\omega-\omega^{\prime}\right) \frac{1}{\pi} \int_{0}^{\infty} C_{\alpha}(\mathbf{Q}, t) \cos \left(\omega^{\prime} t\right) d t
$$

Les deux fonctions EISF et ICF sont très importantes pour la caractérisation et l'analyse des mouvements des atomes d'hydrogènes, et donc de la dynamique de la protéine: $A_{0 \alpha}(Q)$ décrit la structure spatiale du potentiel dans lequel se font les mouvements des particules, et $C_{\alpha}(Q, t)$ contient toute l'information concernant la dynamique de relaxation dans ce potentiel. Il est clair que $C_{\alpha}(Q, 0)=1$ et $C_{\alpha}(Q, t) \rightarrow 0$ lorsque $t \rightarrow \infty$. Dans la limite de $Q \rightarrow 0$, la fonction $C_{\alpha}(Q, t)$ se réduit à la fonction de corrélation des positions d'atomes d'hydrogènes définie comme suit,

$$
\lim _{Q \rightarrow 0} C_{\alpha}(Q, t)=C_{\alpha}(t)=\frac{\left\langle\mathbf{r}_{\alpha}(t) \mathbf{r}_{\alpha}(0)\right\rangle-\left\langle\mathbf{r}_{\alpha}\right\rangle^{2}}{\left\langle\mathbf{r}_{\alpha}^{2}\right\rangle-\left\langle\mathbf{r}_{\alpha}\right\rangle^{2}} .
$$

Dans la limite opposée de $Q \rightarrow \infty$ (i.e., pour de très petites échelles de longueurs) les limites du système deviennent insignifiantes de telle sorte que les mouvements des particules ne sentent plus le confinement du potentiel. Dans ce cas, $C_{\alpha}(Q, t)$ décroît exponentiellement en fonction temps comme, $C_{\alpha}(Q, t)=\exp \left\{-\Gamma_{\alpha}(Q) t\right\}$ où $1 / \Gamma_{\alpha}(Q)$ peut être interpréter comme le temps nécessaire pour les particules de diffuser sur une échelle de longueur de $1 / Q$.

\section{Approximation de Diffusion Gaussienne}

Lorsque le vecteur d'onde diffusé $\mathbf{Q}$ est petit et proche de zéro, l'expression de $I(\mathbf{Q}, t)$ peut se simplifier et, en faisant la moyenne sur toutes les directions angulaires, obtenir:

$$
I(Q, t) \cong \sum_{\alpha=1}^{N} x_{\alpha} \exp \left\{-\frac{Q^{2}}{6}\left\langle\left[\mathbf{r}_{\alpha}(t)-\mathbf{r}_{\alpha}(0)\right]^{2}\right\rangle\right\}=\sum_{\alpha=1}^{N} x_{\alpha} \exp \left\{-\frac{Q^{2}}{3}\left\langle r_{\alpha}^{2}\right\rangle\left[1-C_{\alpha}(t)\right]\right\},
$$

où $\left\langle r_{\alpha}^{2}\right\rangle$ est le déplacement carré moyen à l'équilibre, i.e., $\left\langle r_{\alpha}^{2}\right\rangle=\int r_{\alpha}^{2} P_{\alpha e q}\left(\mathbf{r}_{\alpha}\right) d \mathbf{r}_{\alpha}$ et $C_{\alpha}(t)$ la fonction de corrélation stationnaire des positions définie plus haut. Il est à noter que à cause de l'hétérogénéité dans la dynamique des particules (i.e., $x_{\alpha} \neq 1$ ), l'intensité dans Eq.(2.13) est une combinaison de plusieurs fonctions Gaussiennes, et donc n'est plus une fonction Gaussienne de $Q$ [23]. L'expression dans Eq.(2.11) suggère d'écrire, $S_{\text {inc }}(\mathbf{Q}, \omega)=S_{\text {inc }}^{\mathrm{el}}(\mathbf{Q})+S_{\text {inc }}^{\mathrm{qel}}(\mathbf{Q})$, où $S_{\text {inc }}^{\mathrm{el}}(\mathbf{Q})=S_{\mathrm{inc}}(\mathbf{Q}, \omega=0)=\sum_{\alpha=1}^{N} x_{\alpha} S_{\alpha}^{\mathrm{el}}(\mathbf{Q})$ avec,

$$
S_{\alpha}^{\mathrm{el}}(\mathbf{Q})=\frac{\tau}{2}\left\{A_{0 \alpha}(\mathbf{Q})+\frac{2}{\pi}\left[1-A_{0 \alpha}(\mathbf{Q})\right] \int_{0}^{\infty} d y\left[\frac{\sin (y)}{y}\right] C_{\alpha}(\mathbf{Q}, y \tau)\right\} .
$$

Cette fonction est utilisé expérimentalement pour l'analyse des fluctuations des positions des atomes d'hydrogènes. Nous avons dans l'approximation Gaussienne,

$$
S_{\text {inc }}^{\mathrm{el}}(Q) \simeq \frac{\tau}{2} \sum_{\alpha=1}^{N} x_{\alpha} \exp \left\{-\frac{Q^{2}\left\langle r_{\alpha}^{2}\right\rangle}{3}\left[1-\int_{0}^{\infty} d y\left[\frac{\sin (y)}{y}\right] C_{\alpha}(y \tau)\right]\right\} .
$$

Ainsi, à temps de résolution fini, $S_{\mathrm{inc}}^{\mathrm{el}}(Q)$ contient à la fois $\left\langle r_{\alpha}^{2}\right\rangle$ and $C_{\alpha}(\tau)$. Cette fonction $S_{\text {inc }}^{\mathrm{el}}(Q)$ relaxe éventuellement vers le EISF $I(Q, \infty)$ (qui ne dépend uniquement que des $\left\langle r_{\alpha}^{2}\right\rangle$ ) pour des temps de résolution suffisamment long de telle sorte que tous les $C_{\alpha}(\tau) \rightarrow 0$. Il s'ensuit que le déplacement carré moyen observable, $\left\langle R^{2}\right\rangle$, qui prend en compte les fluctuations de toutes les particules dans le système 
protéique, est donné par:

$$
\begin{aligned}
\left\langle R^{2}\right\rangle & =-\left.3 \frac{d\left\{\ln \left[S_{\text {inc }}^{\mathrm{el}}(Q)\right]\right\}}{d\left(Q^{2}\right)}\right|_{Q=0} \\
& =\sum_{\alpha=1}^{N} x_{\alpha}\{\underbrace{3\left\langle r_{\alpha}^{2}\right\rangle_{\text {DW }}}_{\text {Debye-Waller }}+\left\langle r_{\alpha}^{2}\right\rangle\left[1-\int_{0}^{\infty} d y\left[\frac{\sin (y)}{y}\right] C_{\alpha}(y \tau)\right]\},
\end{aligned}
$$

où le premier terme représente la contribution des mouvements vibrationnels (Debye-Waller) dans l'Eq.(2.7). Comme précédemment, nous omettrons ce terme par soucis de simplification. Il apparait que $\left\langle R^{2}\right\rangle$ contient à la fois l'information sur l'extension spatiale du potentiel (i.e., le EISF) et la fonction de relaxation (i.e., le ICF) des mouvements internes. De manière générale, les proportions $x_{\alpha}$, les déplacements carrés moyens $\left\langle r_{\mathrm{a}}^{2}\right\rangle$ et les temps de corrélations $1 / k_{\alpha}(0)$, sont tous des fonctions de la température et donc seraient susceptible de contribuer à la transition dynamique. De plus, $\left\langle R^{2}\right\rangle$ est une fonction croissante de $\tau$ et les atomes d'hydrogènes dits "immobiles" (ou invisibles par les neutrons) sont caractérisés $\operatorname{par} C_{\alpha}(\tau) \simeq 1$.

\section{DYNAMIQUE INTERNE DES PROTÉINES : MODÈLES CLASSIQUES}

Plusieurs situations physiques d'intérêts comme pour des molécules sur des surfaces, dans les systèmes micellaires ou vésiculaires ou encore des cages structurales où le système peut être décrit par une dynamique de saut ou par un processus de diffusion dans une géométrie de confinement [24-27]. La dynamique de saut entre deux (ou plusieurs) sites et la diffusion dans une sphère représentent les modèles conventionnels les plus simples qui sont largement utilisés dans la littérature pour la description des mouvements des atomes d'hydrogènes dans les protéines. Dans cette section, nous examinons ces deux modèles de dynamique interne pour l'interpretation de la transition dynamique. Dans cette analyse, on considère que tous les atomes d'hydrogènes de la protéine sont dynamiquement équivalents, i.e., $x_{\alpha}=1$.

\subsection{Saut ou Transition entre deux Sites non - Équivalents}

Considérons la dynamique de saut d'une particule entre deux sites ponctuels non - équivalents situés en $\mathbf{r}_{1}=\mathbf{0}$ et en $\mathbf{r}_{2}=\mathbf{a}$. Le potentiel de force moyenne correspondant à cette situation est donné par,

$$
\mathrm{e}^{-\beta U(\mathbf{r})}= \begin{cases}\phi & ; \mathbf{r}=\mathbf{0}, \\ 1-\phi & ; \mathbf{r}=\mathbf{a}, \quad ; \phi=\frac{\tau_{1}}{\tau_{1}+\tau_{2}} . \\ 0 & ; \text { ailleurs },\end{cases}
$$

où $\tau_{1}$ and $\tau_{2}$ sont les temps moyens de résidence de la particule dans chaque site. A cause de la symétrie sphérique du problème, il s'agit en fait d'une dynamique de sauts entre un point situé à l'origine et la surface d'une sphere de centre origine et rayon $a$. En réalité, la situation physique de ce problème correspond à une dynamique de transition entre deux minima d'un potentiel séparés par une barrière de potentiel comme montré dans la Fig. 1.

Lorsque $\Delta \gg \mathrm{k}_{\mathrm{B}} \mathrm{T}$, les deux puits sont bien distincts, les mouvements dans chaque puit de potentiel se font essentiellement au voisinage du minimum correspondant et la probabilité de trouver la particule en dehors de ce voisinage est négligeable. Dans ce cas, le problème de transition dans un double puits se réduit à celui de transition entre deux sites ponctuels et on peut définir la probabilité de trouver la particule sur le site 1 par: $\phi=1 /[1+\exp \{-\beta \Delta G\}]$, où $\Delta G$ représente la variation d'énergie standard de la transition entre les deux sites. 

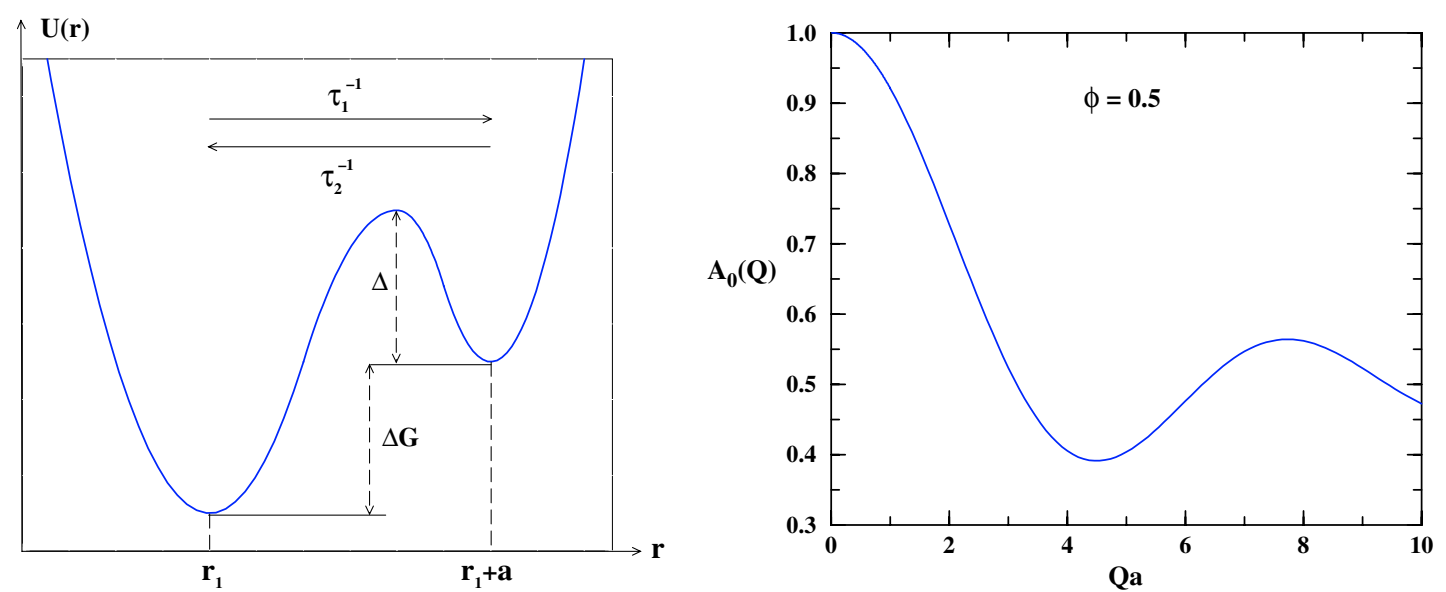

Figure 1. Potentiel double puits et facteur de forme (EISF) pour la dynamique de saut entre deux sites.

Le EISF et le ICF (ou encore la fonction de relaxation des fluctuations des positions de la particule) pour cette dynamique sont donnés par les relations,

$$
A_{0}(Q)=1-2 \phi(1-\phi)\left[1-j_{0}(Q a)\right] \text { and } C(t)=\mathrm{e}^{-\Gamma t} ; \Gamma=\tau_{1}^{-1}+\tau_{2}^{-1},
$$

où $j_{0}(z)$ est la fonction de Bessel sphérique de première espèce et d'ordre zero. Le EISF ne s'annule jamais mais oscille autour de la valeur $1-2 \phi(1-\phi)$. Le déplacement carré moyen observable est alors,

$$
\left\langle R^{2}\right\rangle=\phi(1-\phi) a^{2}\left[1-\frac{2}{\pi} \arctan \left(\frac{1}{\Gamma \tau}\right)\right],
$$

avec $\tau$ étant le temps de résolution expérimentale. Dans cette expression, les variables dépendant de la température sont $\phi$ et $\Gamma \sim \mathrm{e}^{-\beta \Delta}\left[1+\mathrm{e}^{-\beta \Delta G}\right]$. C'est ce modèle (plus la contribution de Debye-Waller mais dans la limite de $\Gamma \tau \gg 1$ ) qui a été utilisé pour étudier les mouvements moléculaires internes et interpréter la transition dynamique dans les protéines [11, 28, 29].

Dans ce modèle, l'origine de la transition dynamique est la suivante: à basse temperature, les positions des particules sont essentiellement localisées autour du minimum du puit de potentiel le plus bas en énergie si bien que l'autre puit n'est presque pas peuplé. Dans ce cas, la variation de $\left\langle R^{2}\right\rangle$ en fonction de la température est donnée par la contribution vibrationnelle (Debye-Waller). Par contre l'élévation de la température entraîne, par sauts au dessus de la barrière énergétique, une augmentation de la population dans le puit de potentiel de haute énergie et distant du puit initial. Ce processus de sauts au dessus de la barrière énergétique se traduit par une croissance non - linéaire de $\left\langle R^{2}\right\rangle$ en fonction de la température.

\subsection{Diffusion à l'intérieur d'une Sphère}

En comparaison au modèle de sauts entre sites, le modèle de diffusion dans une sphère est le modèle le plus simple qu'on puisse utiliser pour la dynamique des atomes d'hydrogènes. Il présente l'avantage d'être caractérisé par un seul paramètre spatial (la taille de la sphère) et un seul temps caractéristique (le temps de diffusion dans la sphère); ce qui représente un atout majeur par rapport aux autres modèles pour l'analyse des données expérimentales. Le potentiel de force moyenne pour une particule effectuant un mouvement de diffusion à l'intérieur d'une sphère imperméable de rayon $a$ s'écrit,

$$
U(r)=\left\{\begin{array}{ccc}
0 & ; & r \leq a \\
\infty & ; & r>a
\end{array}\right.
$$



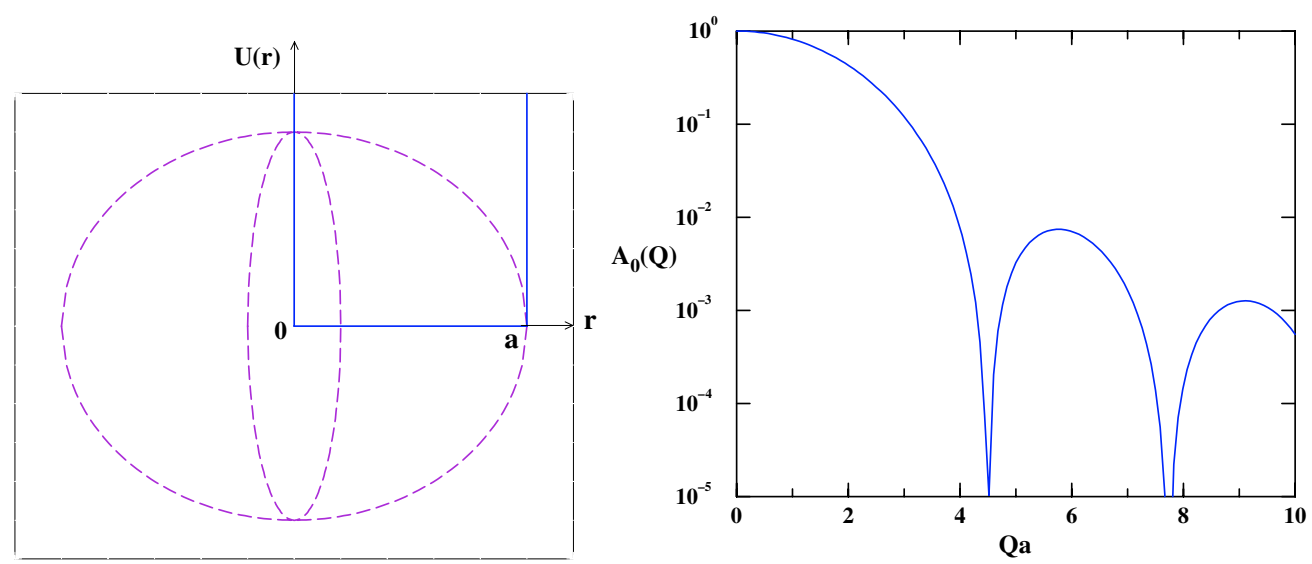

Figure 2. Potentiel de force moyenne et facteur de forme (EISF) pour la diffusion dans une sphere impermeable.

Les EISF et ICF correspondant à cette dynamique sont donnés par les relations [22, 24],

$$
A_{0}(Q)=\left|\frac{3 j_{1}(Q a)}{Q a}\right|^{2} \quad ; \quad C(Q, t)=\sum_{n, l}^{\infty}\left[\frac{A_{n}^{l}(Q)}{1-A_{0}(Q)}\right] \exp \left\{-\Gamma_{n}^{l} t\right\} .
$$

où les $A_{n}^{l}(Q)$ sont donnés par [22, 24],

$$
A_{n}^{l}(Q)=\frac{6(2 l+1)\left(x_{n}^{l}\right)^{2}}{\left(x_{n}^{l}\right)^{2}-l(l+1)}\left[\frac{Q a j_{l+1}(Q a)-l j_{l}(Q a)}{(Q a)^{2}-\left(x_{n}^{l}\right)^{2}}\right]^{2}
$$

et les taux de relaxation, $\Gamma_{n}^{l}=a^{2} /\left(x_{n}^{l}\right)^{2} D$, avec $D$ la constante de diffusion de la particule et $x_{n}^{l}$ les racines de l'équation, $x_{n}^{l} j_{l+1}\left(x_{n}^{l}\right)=l j_{l}\left(x_{n}^{l}\right)\left(j_{l}(z)\right.$ est la fonction de Bessel sphérique de première espèce et d'ordre l). A la différence de la dynamique de sauts, ici le EISF s' annule plusieurs fois comme le montre la Fig. 2. Pour de petites ou grandes valeurs de $Q$, le ICF peut être approximé par une exponentielle,

$$
C(Q, t) \simeq \exp \{-k(Q) t\} \quad ; \quad k(Q) \simeq \begin{cases}\Gamma_{0}^{1}=13 D / 3 a^{2} & ;(Q a)^{2} \leq 5 \\ Q^{2} D & ;(Q a)^{2}>10\end{cases}
$$

où $k(Q)$ est le taux ou la vitesse de relaxation (Fig. 3).

Les déplacements carrés moyens observables pour le modèle de diffusion dans une sphère imperméable sont,

$$
\left\langle R^{2}\right\rangle=\frac{3 a^{2}}{5}\left[1-\frac{2}{\pi} \arctan \left(\frac{1}{\Gamma_{d} \tau}\right)\right] ; \Gamma_{d}=13 D / 3 a^{2},
$$

avec $\tau$ étant le temps de résolution expérimentale et $1 / \Gamma_{d}$ est le temps typique pour la particule de diffuser à travers toute la sphère. A cause de sa simplicité, ce modèle a été utilisé par plusieurs auteurs [26, 30, 31] pour étudier, par exemple, la dynamique interne, la structure et la dynamique des molécules de surface dans les protéines. Pour adapter ce modèle à la transition dynamique, on convient de considérer que le rayon " $a$ " de la sphere diffusion varie avec la température. Par exemple, il suffirait de remplacer $a^{2}$ dans Eq.(3.8) par $\phi(1-\phi) a^{2}$ pour avoir une expression similaire à celle dans Eq.(3.3). Ici, la transition dynamique peut être interprétée comme une augmentation (dilatation activée) non - linéaire en fonction de la température de la taille de la sphère (ou cage structurale) de diffusion. 


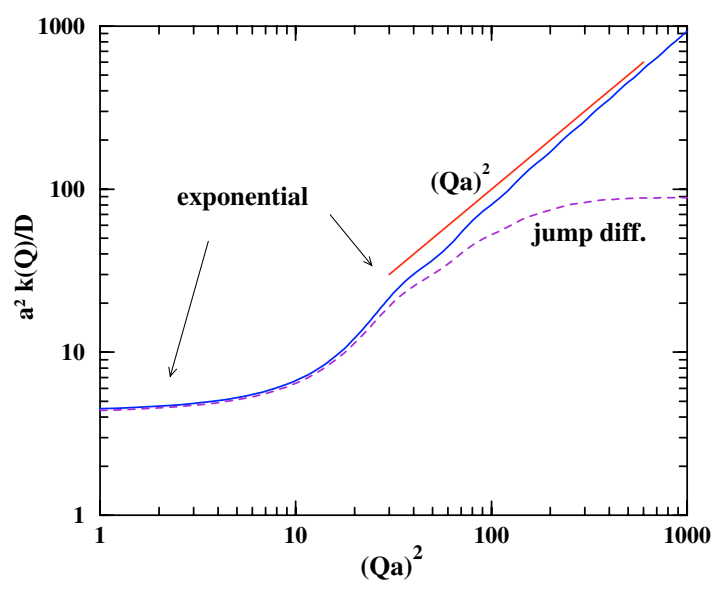

Figure 3. Taux de relaxation réduit en fonction de $(Q a)^{2}$.

\section{EXTENSIONS DES MODÈLES CLASSIQUES : HÉTÉROGÉNÉITÉ DYNAMIQUE}

Jusqu'ici, nous avons considéré des systémes protéiques dynamiquement homogènes dans lesquels les atomes d'hydrogènes ont de mouvements identiques dans un potentiel de force moyenne identique. L'hétérogénéité dynamique consiste à considérer que tous les atomes d'hydrogènes ne sont pas assujettis aux mêmes dynamiques, i.e. nature des mouvements identiques mais dans des potentiels de force moyenne différents, ou encore nature des mouvements et potentiels de force moyenne différents. Le modèle le plus simple du point de vue de la réflexion mais le plus complexe du point de vue de l'analyse serait de considérer chaque atome d'hydrogène du système protéique comme obéissant à sa propre dynamique (e.g., diffusion dans une sphère ou sauts au dessus d'une barrière de potentiel).

Dans cette section nous allons examiné deux modèles simples d'hétérogénéité dynamique construit à partir de la combinaison des deux modèles classiques présentés ci-dessus, à savoir le modèle de diffusion dans une sphère imperméable et la dynamique de sauts au dessus d'une barrière de potentiel [32].

\subsection{Dynamiques Additive et Multiplicative [32]}

\subsubsection{Dynamique Additive}

Considérons la situation où le système protéique peut être subdivisé en deux sous systèmes dont l'un est formé d'une proportion $x$ d'atomes d'hydrogènes effectuant une dynamique de sauts au dessus d'une barrière de potentiel séparant deux sites distant de $a$ et l'autre proportion $(1-x)$ d'atomes d'hydrogénes restante décrivant un processus de diffusion dans une sphère imperméable de rayon $R$. Dans ce cas, on montre que les EISF et ICF pour une telle dynamique sont donnés par les sommes incohérentes,

$$
A_{0}(Q)=x A_{0 j}(Q)+(1-x) A_{0 d}(Q) ; C(Q, t)=x C_{j}(t)+(1-x) C_{d}(Q, t)
$$

où les indices "j" et "d" font respectivement référence à jump (pour la dynamique de sauts) et à diffusion, $A_{0 j}(Q)\left(C_{0 j}(t)\right)$ et $A_{0 d}(Q)\left(C_{d}(Q, t)\right)$ sont respectivement les EISF (ICF) pour la dynamique de sauts au dessus d'une barrière et la diffusion dans une sphère imperméable donnés dans les Eqs.(3.2) et (3.5) (où $a$ est remplacé par $R$ ). La Fig. 4 donne une illustration du facteur de forme pour une telle dynamique. 

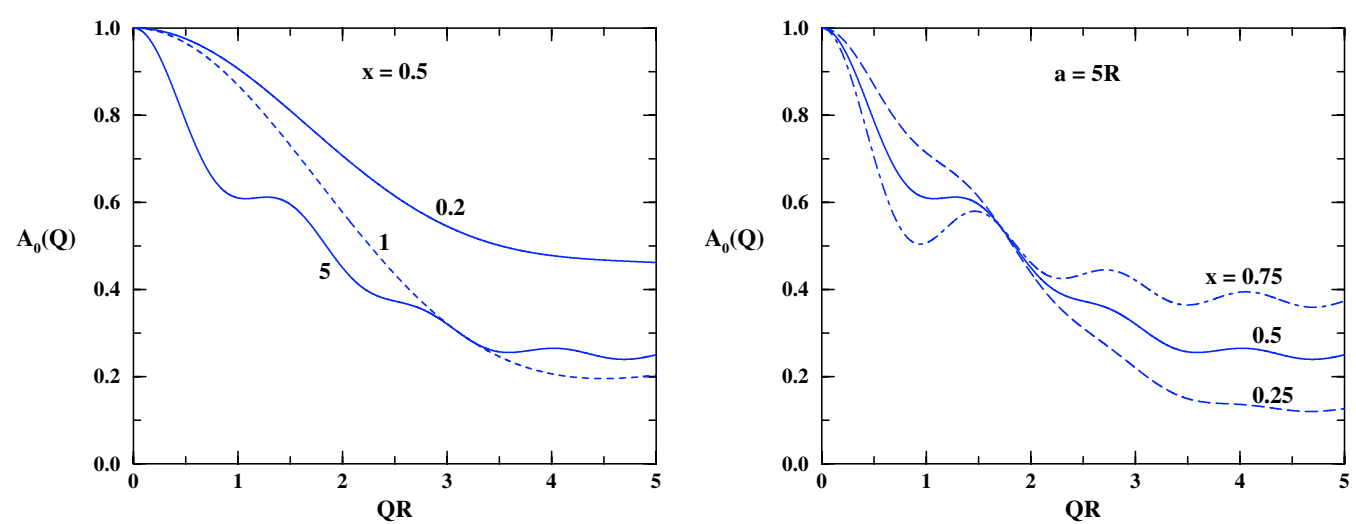

Figure 4. Facteur de forme (EISF) pour la dynamique additive correspondant à $\phi=0.5$. Les chiffres dans la figure de gauche représentent le rapport $a / R$, et la fraction $x$ dans la figure de droite.

\subsubsection{Dynamique Multiplicative}

Un autre exemple d'hétérogénéité dynamique est de considérer un système homogène dans lequel les mouvements des particules sont composites comme suit, $\mathbf{r}(t)=\mathbf{r}$ (dynamique de sauts) + $\mathbf{r}$ (diffusion dans une sphère). Dans ce cas, le EISF est donné par le produit, $A_{0}(Q)=A_{0 j}(Q) A_{0 d}(Q)$ et illustré sur la Fig. 5, et le ICF par,

$$
C(Q, t)=\frac{\overbrace{\left\{A_{0 j}(Q)+\left[1-A_{0 j}(Q)\right] C_{j}(t)\right\}}^{I_{j}(Q, t)} \overbrace{\left\{A_{0 d}(Q)+\left[1-A_{0 d}(Q)\right] C_{d}(t)\right\}}^{I_{d}(Q, t)}-\overbrace{A_{0 j}(Q) A_{0 d}(Q)}^{A_{0}(Q)}}{1-A_{0}(Q)}
$$

où les $A_{0 j}(Q)\left(C_{0 j}(t)\right)$ et $A_{0 d}(Q)\left(C_{d}(Q, t)\right)$ sont définis comme précédemment.

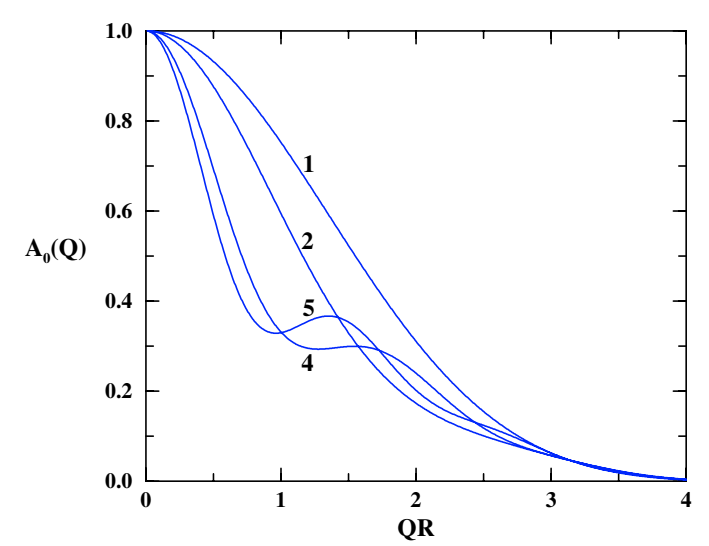

Figure 5. Facteur de forme (EISF) pour la dynamique multiplicative correspondant à $\phi=0.5$. Les chiffres dans la figure représentent le rapport $a / R$. 


\subsubsection{Comportements limites des Dynamiques Additive et Multiplicative}

La fonction de corrélation des positions (ou encore le ICF à $Q \rightarrow 0$ ) pour les deux dynamiques additive et multiplicative peut s'écrire de maniére compacte comme suit,

$$
C(0, t)=c \mathrm{e}^{-\Gamma_{j} t}+(1-c) \mathrm{e}^{-\Gamma_{d} t} \quad ; \quad c=\left\{\begin{array}{c}
x \\
{\left[1+\frac{3 R^{2}}{5 \phi(1-\phi) a^{2}}\right]^{-1} ; \text { additive }}
\end{array}\right.
$$

où $c$ joue maintenant le rôle de fraction effective. On peut constater que $c$ est une fonction de la température à travers $\phi$ dans le cas de la dynamique multiplicative. Comme le montre la Fig. 6, les fluctuations des positions relaxent soit bi-exponentiellement ou exponentiellement dépendant de la fraction $c$ et du rapport des temps de relaxation respectivement à la dynamique de sauts et de la diffusion. Néanmoins, nous avons les comportements limites suivants:

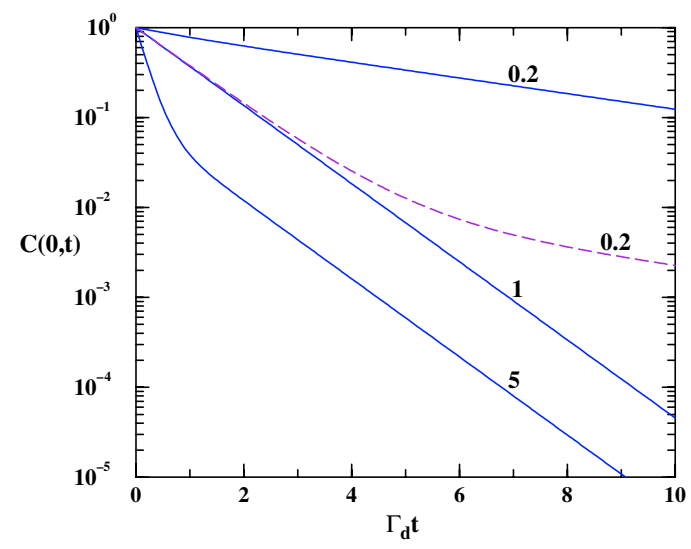

Figure 6. $C(0, t)$ pour différentes valeurs du rapport $\Gamma_{j} / \Gamma_{d}=0.2,1,5$ comme montré sur la figure. Les courbes continues correspondent à $c=0.91$ (i.e., à $x=0.91$ pour la dynamique additive, et à $\phi=0.5$ et $a=5 R$ pour la dynamique multiplicative) tandis que la courbe en pointillé à $c=0.016$ (i.e., à $x=0.016$ pour la dynamique additive, et à $\phi=0.5$ et $a=R / 5$ pour la dynamique multiplicative).

- limites sur les échelles de longueurs $Q$ :

$$
\begin{gathered}
Q \rightarrow 0: C(Q, t)=\left\{\begin{array}{l}
\text { bi-exponentielle } ; \text { additive } \\
\text { bi-exponentielle ; multiplicative }
\end{array}\right. \\
Q \rightarrow \infty: C(Q, t)=\left\{\begin{array}{l}
\text { bi-exponentielle } ; \text { additive } \\
\text { exponentielle } ; \text { multiplicative }
\end{array}\right.
\end{gathered}
$$

- limites sur les échelles de temps:

$$
\begin{gathered}
\left(R, \Gamma_{d}\right) \rightarrow 0: \text { processus }= \begin{cases}\text { sauts }+\mathrm{H} \text { immobiles } ; & \text { additive } \\
\text { sauts } ; \text { multiplicative }\end{cases} \\
\left(a, \Gamma_{j}\right) \rightarrow 0: \text { processus }= \begin{cases}\text { diffusion }+\mathrm{H} \text { immobiles } ; & \text { additive } \\
\text { diffusion } & ; \text { multiplicative }\end{cases}
\end{gathered}
$$


De même, nous avons des relations simples en ce qui concerne les déplacements carrés moyens,

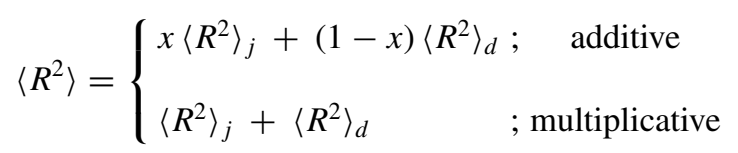

où $\left\langle R^{2}\right\rangle_{j}$ et $\left\langle R^{2}\right\rangle_{d}$ sont respectivement donnés par les Eqs.(3.3) et (3.8).

\subsection{Modèle en Couche des Dynamiques Additive et Multiplicative}

En guise de généralisation sur les deux modèles d'hétérogénéité dynamique présenté ci-dessus, nous considérons un modèle en couche où la protéine de diamètre $d$ peut être subdivisée en trois couches dynamiques emboîtées de diamètres $d_{1}, d_{2}$ et $d$, tel que $0 \leq d_{1} \leq d_{2} \leq d$. La première couche dynamique de diamètre $d_{1}$ constitue le coeur de la protéine, la deuxième couche comprise entre les volumes de diamètres $d_{1}$ et $d_{2}$ forme la partie intermédiaire, et la troisième couche la plus extérieure forme la couronne de surface en contact avec l'environnement extérieur de la protéine. Nous supposons que les atomes d'hydrogènes du coeur ( 1 ère couche) de la protéine effectuent un mouvement de diffusion à l'intérieur d'une sphère de rayon $R_{1}$, ceux de la zone intermédiaire un movement composite résultant de la combinaison de la diffusion à l'intérieur d'une sphère de rayon $R_{2}$ et d'une dynamique de sauts entre deux sites distants de $a_{2}$, et les atomes périphériques de la couche trois une dynamique de sauts entre deux sites distants de $a_{3}$. De manière générale, les diamètres $d_{1}, d_{2}$ et $d$, sont fonction de la température et des tensions superficielles entre les surfaces en contact. Pour ce modèle, la fonction intermédiaire d'intensité diffusée incohérente s'écrit:

$$
I(\mathbf{Q}, t)=\underbrace{\underbrace{x_{1} I_{1}(\mathbf{Q}, t)}_{\text {diffusion }-R_{1}}+\underbrace{x_{3} I_{3}(\mathbf{Q}, t)}_{\text {sauts }-a_{3}}}_{\text {additive }}+\underbrace{\underbrace{x_{2} I_{2}(\mathbf{Q}, t)}_{\text {diffusion }-R_{2} \text { et sauts }-a_{2}}}_{\text {multiplicative }}
$$

où les proportions $x_{i}$ d'atomes d'hydrogènes dans chaque couche sont définies (pour une distribution isotrope des atomes d'hydrogènes dans la protéine) par,

$$
\underbrace{x_{1}=\int_{0}^{d_{1} / 2} 4 \pi r^{2} g(r) d r}_{\text {couche } 1} ; \underbrace{x_{2}=\int_{d_{1} / 2}^{d_{2} / 2} 4 \pi r^{2} g(r) d r}_{\text {couche } 2} ; \underbrace{x_{3}=1-x_{1}-x_{2}}_{\text {couche } 3} .
$$

Dans ces expressions, $g(r)$ (tel que $\int_{0}^{d / 2} 4 \pi r^{2} g(r) d r=1$ ) désigne la densité de probabilité de trouver un atome d'hydrogène à la distance $r$ du barycentre de l'ensemble des atomes d'hydrogènes du système protéique considéré. La fonction $g(r)$ pour une protéine peut être directement calculée à partir des coordonnées de la structure du système protéique. Toutefois, deux choix simples sont possibles:

$$
g(r)= \begin{cases}6 /\left(\pi d^{3}\right) & ; \text { dist. homogène } \\ \rho \exp \left(-4 \pi r^{3} \rho / 3\right) /\left[1-\exp \left(-\pi d^{3} \rho / 6\right)\right] & ; \text { dist. aléatoire }\end{cases}
$$

pour $0 \leq r \leq d / 2$ et où $\rho$ représente le nombre d'atomes d'hydrogènes par unité de volume.

On peut rajouter des détails supplémentaires en supposant que dans chaque couche dynamique de la protéine, la taille des sphères de diffusion et le distance entre sites pour la dynamique de sauts admettent une distribution [33]. Dans le cas des distributions discrètes, on peut alors dans l'Eq. (4.9) faire le changement suivant,

$$
x_{i} I_{i}(\mathbf{Q}, t) \longrightarrow \sum_{j} x_{i j} I_{i j}(\mathbf{Q}, t)
$$


où $x_{i j}$ (tel que $\sum_{j} x_{i j}=x_{i}$ ) représente la proportion d'atomes d'hydrogènes de la couche $i$ effectuant, selon le type de dynamique, un mouvement de diffusion dans une sphère de rayon $R_{i j}$ ou une dynamique de sauts entre deux sites séparés de $a_{i j}$, et $I_{i j}(\mathbf{Q}, t)$ est la fonction intermédiaire d'intensité diffusée incohérente associée. Les déplacements carrés moyens pour une telle dynamique s'écrit,

$$
\left\langle R^{2}\right\rangle=\frac{3 R_{\mathrm{e}}^{2}}{5}\left[1-\frac{2}{\pi} \arctan \left(\frac{1}{\Gamma_{\mathrm{ed}} \tau}\right)\right]+\phi_{\mathrm{e}}\left(1-\phi_{\mathrm{e}}\right) a_{\mathrm{e}}^{2}\left[1-\frac{2}{\pi} \arctan \left(\frac{1}{\Gamma_{\mathrm{ej}} \tau}\right)\right],
$$

où le rayon effectif $R_{\mathrm{e}}$, la distance effective $a_{\mathrm{e}}$ entre sites et la population effective $\phi_{\mathrm{e}}$ sont définis par,

$$
\left\{\begin{array}{l}
R_{\mathrm{e}}^{2}=\sum_{i k} x_{i k} R_{i k}^{2} \\
\phi_{\mathrm{e}}\left(1-\phi_{\mathrm{e}}\right) a_{\mathrm{e}}^{2}=\sum_{i k} x_{i k} \phi_{i k}\left(1-\phi_{i k}\right) a_{i k}^{2}
\end{array}\right.
$$

et les taux de relaxation effectifs pour la diffusion $\Gamma_{\text {ed }}$ et pour la dynamique de sauts $\Gamma_{\text {ej }}$ sont obtenus à partir de,

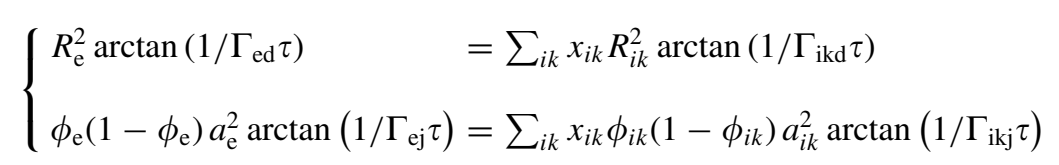

Il vient de ce qui précède que les paramètres $R_{\mathrm{e}}, a_{\mathrm{e}}, \phi_{\mathrm{e}}, \Gamma_{\mathrm{ed}}$ et $\Gamma_{\mathrm{ej}}$ sont des fonctions non-linéaires de la température, et que $\Gamma_{\text {ed }}$ et $\Gamma_{\text {ej }}$ dépendent en plus du temps de résolution expérimental. Ce modèle illustre la difficulté d'interpréter les résultats expérimentaux en termes de transition dynamique. En effet, il apparaît clairement de cette description que la variation non-linéaire des déplacements carrés moyens peuvent être de plusieurs origine. Ce modèle indique aussi que à cause de l'hétérogénéité dynamique intrinsèque de la protéine, il est même plausible pour la transition dynamique d'utiliser le modèle de diffusion à l'intérieur d'une sphère mais en permettant le rayon de varier avec la température. Par ailleurs, un modèle si détaillé devient très vite riche en paramètres et, de ce fait, perd son intérêt car il devient impossible, du point de vue pratique, de (chercher à) déterminer tous les paramètres des Eqs.(4.14) et (4.15).

\section{MODÈLE DE DIFFUSION À L'INTÉRIEUR DE DEUX SPHÈRES CONCENTRIQUES}

Dans les sections précédentes, nous avons essentiellement discuté deux modèles classiques de dynamique (à savoir, la diffusion à l'intérieur d'une sphère et la dynamique de sauts entre deux sites ponctuels non - équivalents) ayant des caractéristiques différentes tant sur le EISF que sur le ICF. Ensuite, nous avons examiné des combinaisons de ces deux modèles dans le cadre de l'hétérogénéité dynamique. Dans cette section, nous présentons le modèle de diffusion à l'intérieur de deux sphères concentriques [22]. Dans ce modèle, on considère la dynamique de transition entre deux régions séparés par une barrière de potentiel de hauteur $\varepsilon$. La région 1 est une sphère de rayon $a$ d'énergie $-\varepsilon$ et la région 2 une couronne sphérique d'épaisseur radiale $R-a$ (comprise entre deux sphères concentriques de rayon $a$ et $R$, avec $R>a$ ) d'énergie nulle; la dynamique dans chacune des régions étant décrite par une processus de diffusion de constante $D$. Par construction, ce modèle intègre tous les ingrédients et caractéristiques de la diffusion à l'intérieur d'une sphere et de la dynamique de sauts entre deux sites non - équivalents. De plus, il a l'avantage d'être analytiquement soluble. L'analyse détaillée de ce modèle est présentée dans la Ref. [22]. Nous allons nous contenter ici de donner les expressions de quelques fonctions importantes dans le cadre de cette discussion. 

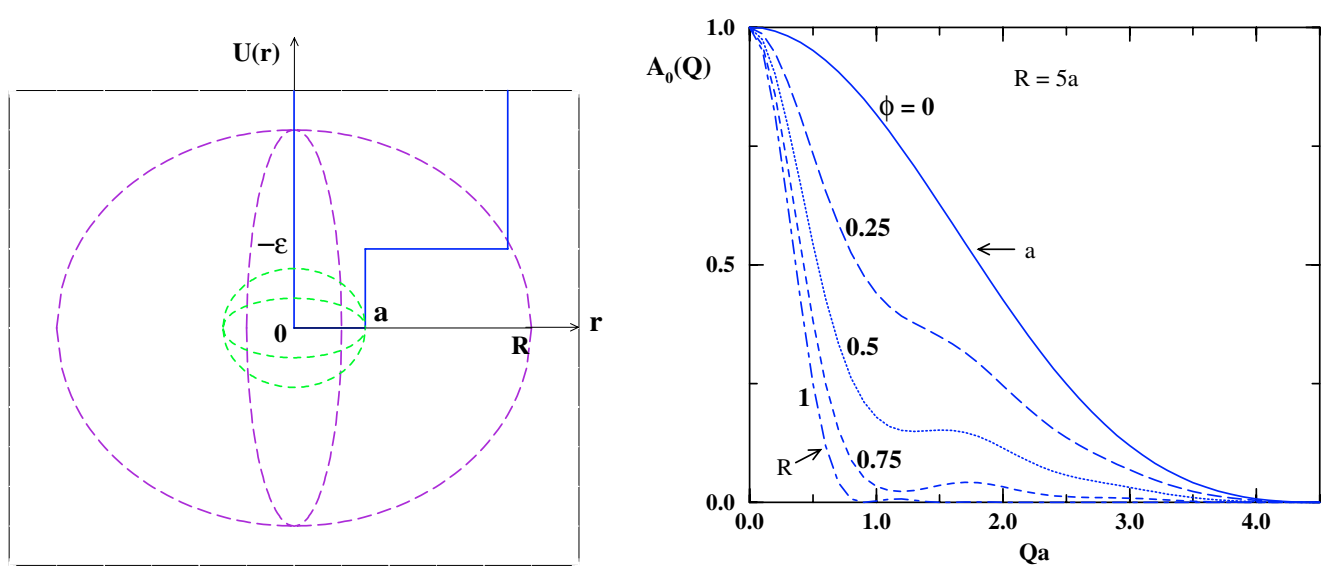

Figure 7. Potentiel de force moyenne et facteur de forme (EISF) pour la diffusion à l'intérieur de deux spheres concentriques. Les valeurs des proportions sont: $\phi \rightarrow 1-\phi\left[1-(a / R)^{3}\right]$.

Le potentiel de force moyenne pour une particule effectuant un mouvement de diffusion à l'intérieur de deux sphères concentriques de rayon $a$ et $R(R>a)$ s'écrit (voir Fig.7),

$$
U(r)=\left\{\begin{array}{cl}
-\varepsilon ; & 0 \leq r \leq a, \\
0 ; & a<r \leq R, \\
\infty ; & r>R .
\end{array}\right.
$$

Le EISF correspondant à ce potentiel est une somme cohérente des amplitudes de diffusion dans une sphère et dans une couronne entre deux sphères concentriques [22],

$$
A_{0}(Q)=|\phi \underbrace{\left[\frac{3 j_{1}(Q a)}{Q a}\right]}_{\text {sphère }}+(1-\phi) \underbrace{\left\{\frac{R^{3}\left[\frac{3 j_{1}(Q R)}{Q R}\right]-a^{3}\left[\frac{3 j_{1}(Q a)}{Q a}\right]}{R^{3}-a^{3}}\right\}}_{\text {couronne sphérique }}|^{2},
$$

où $\phi$, le degré de perméabilité représentant la proportion de la population des particules qui diffusent uniquement dans la sphère intérieur de rayon $a$, est défini par

$$
\phi=\frac{1}{1+\exp \{-\beta \Delta G\}} ; \Delta G=\varepsilon-\mathrm{T} \Delta S \text { et } \Delta S=\mathrm{k}_{\mathrm{B}} \ln \left(\frac{R^{3}-a^{3}}{a^{3}}\right),
$$

avec $\Delta S$ et $\Delta G$ étant respectivement la variation d'entropie et d'énergie standard entre les deux régions. Comme pour la dynamique de sauts entre de sites, ici aussi nous avons $\phi=\tau_{1} /\left(\tau_{1}+\tau_{2}\right)$ où $\tau_{1}$ et $\tau_{2}$ sont les temps de résidences respectivement dans la sphère intérieur (région 1) et la couronne sphérique extérieur (région 2) [22]. Noter que,

$$
\phi=\left\{\begin{array}{cl}
1 & ; \beta \varepsilon \rightarrow \infty, \\
1 / 2 & ; T=T_{\mathrm{m}}=\varepsilon / \Delta S, \\
(a / R)^{3} & ; \beta \varepsilon \rightarrow 0
\end{array}\right.
$$

Lorsque $\phi=1$ (ou $\phi=0$ ), l'Eq. (5.2) se réduit au EISF de la diffusion à l'intérieur d'une sphère de rayon $a$ (ou d'une couronne sphérique d'épaisseur $R-a$ ). Comme le montre la Fig. 7, le EISF présente en fonction de $\phi$ un comportement mixte entre la diffusion dans une sphère et la dynamique de sauts 

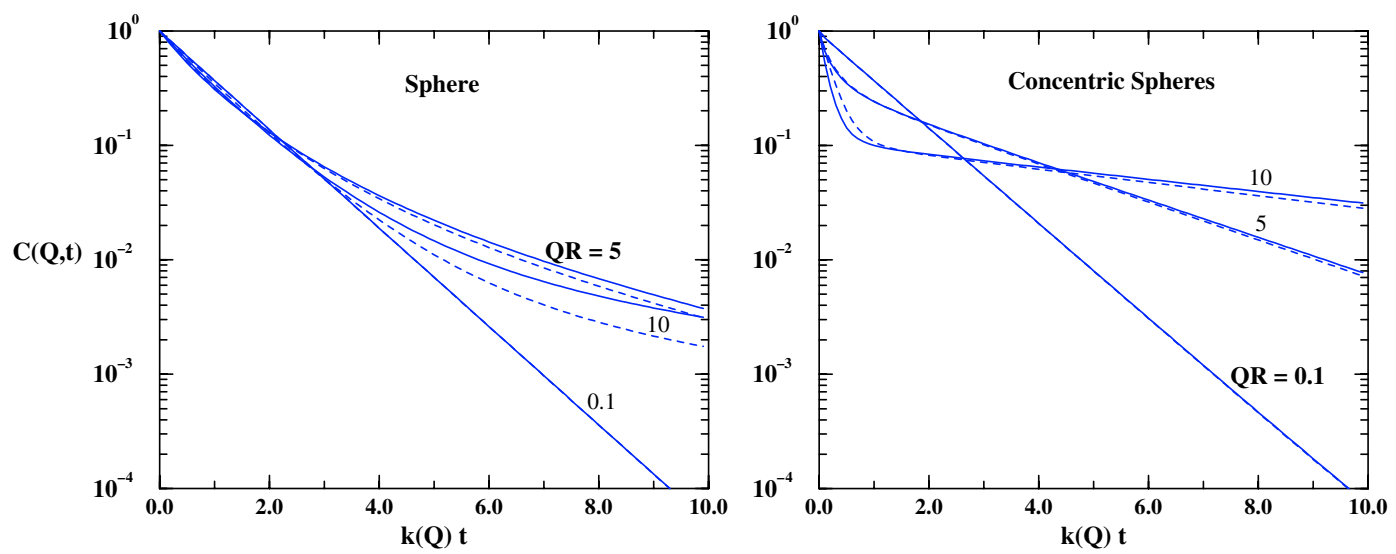

Figure 8. Fonctions de corrélation à l'intérieur d'une sphère de rayon $R$ et de deux sphères concentriques avec $R=5 a$ et $\phi=0.5$. Les courbes en pointillé correspondent au processus de diffusion par sauts [22].

entre deux sites. Le ICF correspondant à cette dynamique est donné par la double exponentielle [22],

$$
C(Q, t)=\left(\frac{A_{0}^{1}(Q)}{1-A_{0}(Q)}\right) \exp \left\{-\Gamma_{0}^{1} t\right\}+\left(\frac{A_{1}^{0}(Q)}{1-A_{0}(Q)}\right) \exp \left\{-\Gamma_{1}^{0} t\right\}
$$

où les facteurs $A_{0}^{1}(Q)$ et $A_{1}^{0}(Q)$ sont définis dans Ref. [22]. $1 / \Gamma_{0}^{1} \propto f(a / R) D / R^{2}$ est le temps typique pour la particule de diffuser dans toute la grande sphère (i.e., à la fois dans la sphère et la couronne sphérique) et $\Gamma_{1}^{0}=\tau_{1}^{-1}+\tau_{2}^{-1}$ est le taux de relaxation des populations entre les deux regions (comme dans la dynamique de sauts entre deux sites). Le tableau 1 donne quelques valeurs de $\Gamma_{0}^{1}$ et $\Gamma_{1}^{0}$ en fonction de $\phi$, et la Fig. 8 illustre la transition exponentielle - bi-exponentielle du ICF en fonction du temps.

Table 1. Taux de relaxations.

\begin{tabular}{l|cc}
\hline \hline & $\phi=0.5\left[1+(a / R)^{3}\right]$ & $\phi=(a / R)^{3}$ \\
\hline$R^{2} \Gamma_{0}^{1} / D$ & 4.539 & 4.333 \\
$R^{2} \Gamma_{1}^{0} / D$ & 1.756 & 20.191 \\
\hline \hline
\end{tabular}

Les déplacements carrés moyens sont donnés par,

$$
\begin{aligned}
\left\langle R^{2}\right\rangle & =\frac{3}{5}\left[\phi a^{2}+(1-\phi)\left(\frac{R^{5}-a^{5}}{R^{3}-a^{3}}\right)\right]\left[1-\frac{2}{\pi} \arctan \left(\frac{1}{\Gamma_{0}^{1} \tau}\right)\right], \\
& =p(1-p) a_{\mathrm{eff}}^{2}\left[1-\frac{2}{\pi} \arctan \left(\frac{1}{\Gamma_{0}^{1} \tau}\right)\right],
\end{aligned}
$$

où, pour la comparaison avec l'Eq. (3.3), on a définie la distance effective $a_{\mathrm{eff}}^{2}=3\left(\phi a^{2}+R^{2}\right)^{2} /\left(5 \phi R^{2}\right)$ et la fraction $p=\phi R^{2} /\left(\phi a^{2}+R^{2}\right)$. Ici, en plus de la fraction $p$, la distance $a_{\text {eff }}$ devient aussi fonction de la température. Ces expressions (5.6) et (5.7) montrent bien que le modèle de diffusion dans deux sphères concentriques est une extension du modèle de dynamique par sauts discuté plus haut. En effet, lorsque $R \gg a \sqrt{\phi}$, on a $a_{\text {eff }}^{2} \rightarrow 3 R^{2} / 5$ et $p \rightarrow \phi$ comme dans l'Eq. (3.3). 


\section{TRANSITION DYNAMIQUE : CONSTANTES DE FORCE INTERNE D'UNE PROTÉINE}

Nous allons terminer cette présentation en donnant quelques caractéristiques classiques de ce qu'on appelle (communément et abusivement) la transition dynamique. En effet, bien qu'il ne s'agissent pas d'une transition au sens des transitions de phases (transition de premier ordre comme dans la transition liquide - gaz, ou transition du second ordre comme dans la transition paramagnétique) on appelle transition dynamique l'écart au comportement linéaire de la variation des déplacements carrés moyens en fonction de la température. Plusieurs expériences ont mis en évidence l'existence de la transition dynamique dans les systèmes protéiques hydratés autour de $180-200 \mathrm{~K}$ [6-18]. Cette transition traduit un changement activé des états conformationnels de la protéine en fonction de la température. L'image simple qu' on a de la transition dynamique est celle de deux états conformationnels, au moins, correspondant à des puits de potentiel séparés par une barrière de potentiel. A basse température, les mouvements (harmoniques) des atomes de la protéine étant localisés autour du minimum des états conformationnels de plus basses énergies, les déplacements carrés moyens augmentent linéairement avec la température. A haute température, l'amplitude des mouvements (anharmoniques) devient plus grande de sorte à effectuer un franchissement de barrière des états conformationnels de basses énergies vers ceux de hautes énergies. Il en résulte une augmentation non-linéaire des déplacements carrés moyens. La transition dynamique peut donc être résumée comme la transition de mouvements harmoniques vers des mouvements anharmoniques due au franchissement de barrière de potentiel.

Dans ce contexte, Bicout et Zaccai [16] ont formulé une expression simple des déplacements carrés moyens pour décrire la transition dynamique:

$$
\begin{aligned}
\left\langle R^{2}(\mathrm{~T})\right\rangle= & \left\{\phi(\mathrm{T}) \cdot\left\{\frac{\mathrm{k}_{\mathrm{B}} \mathrm{T}}{k_{v}}\left[\frac{\theta}{2 \mathrm{~T}} \operatorname{cotanh}\left(\frac{\theta}{2 \mathrm{~T}}\right)-1\right]+\frac{\mathrm{k}_{\mathrm{B}} \mathrm{T}}{k_{1}}\right\}\right. \\
& \left.+[1-\phi(\mathrm{T})] \cdot \frac{\mathrm{k}_{\mathrm{B}} \mathrm{T}}{k_{2}}\right\}\left\{1-\frac{2}{\pi} \arctan \left[\frac{1}{\Gamma(\mathrm{T}) \tau}\right]\right\},
\end{aligned}
$$

où $\phi(\mathrm{T})=1 /\left[1+\exp \left(-\Delta G / \mathrm{k}_{\mathrm{B}} \mathrm{T}\right)\right]$ est la proportion des mouvements des états conformationnels de basses énergies avec $\Delta G=\Delta H-T \Delta S$ la variation d'énergie standard, $\Delta H$ la différence d'enthalpie et $\Delta S$ la différence d'entropie de conformations entre les deux états. $k_{1}$ and $k_{2}$ sont les constantes de force associées respectivement aux états conformationnels de basses et hautes énergies, $1 / \Gamma(\mathrm{T})$ le temps de relaxations des fluctuations conformationnelles et $\tau$ le temps de résolution expérimental. La Fig. 9 illustre le comportement de $\left\langle R^{2}(\mathrm{~T})\right\rangle$ pour la transition dynamique.

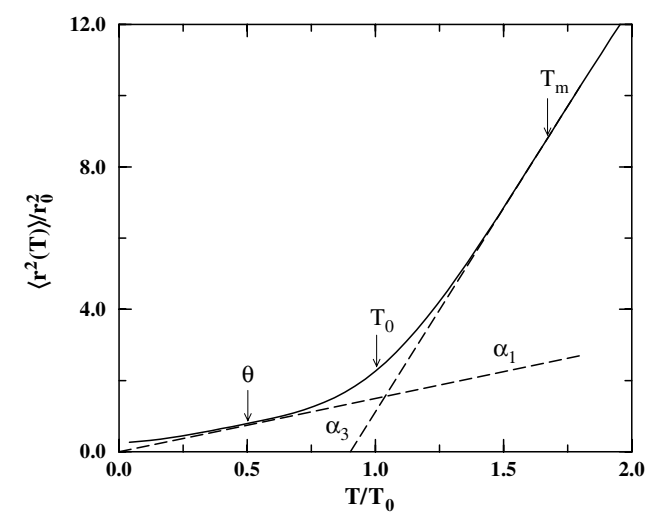

Figure 9. Schéma théorique de la transition dynamique selon l'Eq. (6.1). Les pentes $\alpha_{i}=\mathrm{k}_{\mathrm{B}} / k_{i}$ à basse et haute température sont obtenues à partir de l'Eq. (6.3). $\theta$ est la température de Debye, $\mathrm{T}_{\mathrm{m}}$ la température de compensation, et $\mathrm{T}_{0}$ la température de transition. 
Comme l'écart au comportement linéaire de $\left\langle R^{2}(\mathrm{~T})\right\rangle$ (i.e., mouvements anharmoniques) est contrôlé par la fraction $1-\phi(\mathrm{T})$ des atomes qui franchissent la barrière de potentiel, nous avons définie une température de transition comme suit,

$$
\phi\left(\mathrm{T}_{0}\right)=90 \% \Longleftrightarrow \mathrm{T}_{0}=\frac{\Delta H}{\Delta S+\mathrm{k}_{\mathrm{B}} \ln (9)}, \quad \text { i.e. }, \quad \frac{1}{\mathrm{~T}_{0}}=\frac{1}{\mathrm{~T}_{\mathrm{m}}}+\frac{\mathrm{k}_{\mathrm{B}} \ln (9)}{\Delta H},
$$

où $\mathrm{T}_{\mathrm{m}}$, définie tel que $\phi\left(\mathrm{T}_{\mathrm{m}}\right)=0.5$, est la température à laquelle se fait la compensation entre la variation d'énergie et d'entropie. Par cette définition, la transition dynamique se produit lorsque environ 10\% des atomes commencent à franchir la barrière de potentiel. Pour caractériser la flexibilité interne thermique de la protéine pour des températures $\theta \leq \mathrm{T}<\mathrm{T}_{\mathrm{m}}$, on peut linéariser l'Eq. (6.1) pour obtenir:

$$
\left\langle R^{2}(\mathrm{~T})\right\rangle \simeq \begin{cases}\mathrm{k}_{\mathrm{B}} \mathrm{T} / k_{1} & ; \quad \theta<\mathrm{T}<\mathrm{T}_{0} \\ \mathrm{k}_{\mathrm{B}} \mathrm{T} / k_{3}-\text { cte } & ; \quad \mathrm{T}_{0}<\mathrm{T} \sim \mathrm{T}_{\mathrm{m}}\end{cases}
$$

où $k_{3}$ représente la constante force à $300 \mathrm{~K}$.
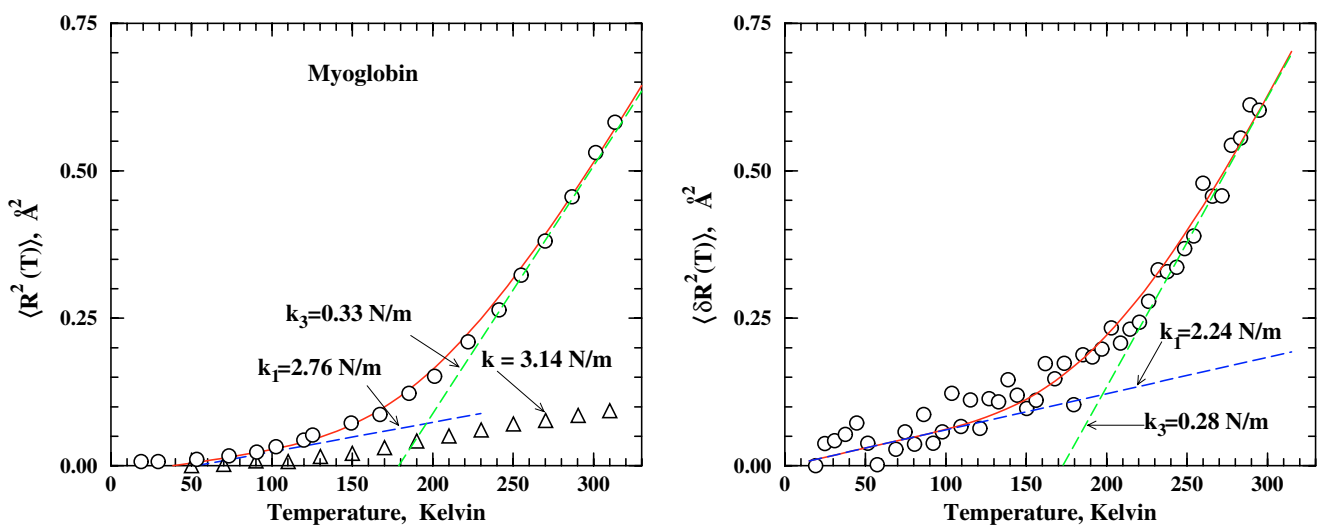

Figure 10. Déplacements carrés moyens en fonction de la température (Transition dynamique) dans la myoglobine (gauche) (de Doster et al. [11]) et dans la bactériorhodopsine (PM)(droite) (de Réat et al. [34]). Les cercles représentent les points expérimentaux et les lignes continues le meilleur ajustement théorique à partir de l'Eq. (6.1) avec les paramètres listés dans le tableau 2. Les triangles représente un exemple d'absence de transition de dynamique pour la myoglobine dans la trehalose (de Cordone et al. [35]).

Cette théorie (dans la limite $\Gamma(\mathrm{T}) \tau \gg 1$ ) a été utilisé pour décrire la transition dynamique et déterminer les énergies et constantes de force associées dans les systémes protéiques [16]. La Fig. 10 montre un exemple de cette application sur la myoglobine et la bactériorhodopsine dans la membrane pourpre (PM) de Halobacterium salinarum [16]. Nous avons trouvé que la transition dynamique est associées à des barrières d'énergies de l'ordre de $R T$ avec des forces de l'ordre de $10-300 \mathrm{pN}$. Cette analyse permet aussi de faire une comparaison rationnelle entre systèmes protéiques (e.g., Fig. 11. Voir aussi Ref. [16] pour une discussion plus détaillée).

\section{CONCLUSION ET PERSPECTIVE}

La dynamique interne des protéines est un processus muldidimensionnel et hétérogène: dynamique muldidimensionnelle où quelques degrés de liberté sont couplés à un environnement fluctuant, et dynamique hétérogène où des classes d'atomes, avec des environnements dynamiques différents, expérimentent des potentiels et mouvements différents. Toute la question est de savoir comment intégrer ces deux aspects dans un modèle simple mais réaliste de la dynamique des protéines. De ce point de vue, la modèlisation et les simulations jouent un rôle majeur et seront décisives dans l'investigation de ces problèmes. 
Table 2. Paramètres correspondant aux courbes théoriques de la Fig. 10. $\Delta H$ et $\Delta S$ sont respectivement les différences d'enthalpie et d'entropie de conformations entre les deux états de la transition. $\Delta G_{0}=\ln (9) \mathrm{RT}_{0}$ et $\Delta G$ représentent respectivement les énergies libres de la barrière de transition à $\mathrm{T}_{0}$ et $300 \mathrm{~K}(\mathrm{RT} \simeq 0.6 \mathrm{kcal} / \mathrm{mol}$ à $300 \mathrm{~K}) . \theta$ est la température de Debye, $\mathrm{T}_{\mathrm{m}}=\Delta H / \Delta S$ la température de compensation, et la température de transition $\mathrm{T}_{0}$ est calculée à partir de Eq. (6.2). $k_{1}$ and $k_{2}$ sont les constantes de force associées respectivement aux états conformationnels de basses et hautes énergies, (cf. Eq. (6.1)), et $k_{3}$ est la constante force à $300 \mathrm{~K}$.

\begin{tabular}{lccc}
\hline \hline & Parameters & Myoglobin & PM wet \\
\hline Energies & $\Delta S(\mathrm{R})$ & 1.80 & 1.65 \\
(kcal/mol) & $\Delta H$ & 1.59 & 1.57 \\
& $\Delta G$ & 0.52 & 0.59 \\
& $\Delta G_{0}$ & 0.88 & 0.90 \\
Températures (K) & $\theta$ & 110 & 110 \\
& $\mathrm{~T}_{0}$ & 200 & 205 \\
& $\mathrm{~T}_{\mathrm{m}}$ & 444 & 480 \\
& & & \\
\hline Constantes & $k_{1}$ & 2.76 & 2.24 \\
de Force $(\mathrm{N} / \mathrm{m})$ & $k_{2}$ & 0.28 & 0.23 \\
& $k_{3}$ & 0.33 & 0.28 \\
\hline \hline
\end{tabular}

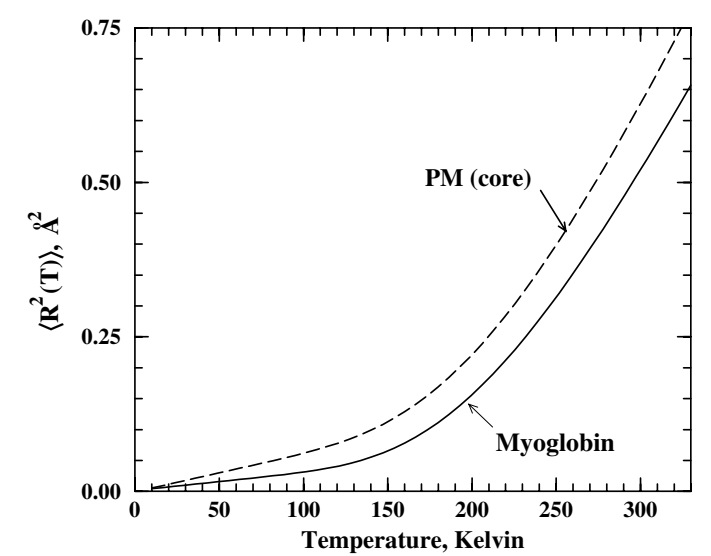

Figure 11. Comparaison de la transition dynamique entre la myoglobine et la PM.

Dans notre discussion, nous avons exposé comment développer des modèles simples et analytiques de dynamique intramoléculaire des protéines qui tiennent compte de la transition dynamique. Ces modèles peuvent être améliorés dans plusieurs directions. Par exemple, on pourrait considérer les effets de plusieurs barrières de potentiel (hiérarchiques) et ceux de l'environnement (solvant) sur la transition dynamique, ou encore développer un modèle qui intégrerait à la fois le transition dynamique et les caractéristiques du repliement des protéines. Ces différents axes feront l'objet de prochaines présentations.

\section{Références}

[1] J. A. McCammon and S. C. Harvey. Dynamics of Proteins and Nucleic Acids, (Cambridge University Press, New York, 1987).

[2] C. L. Brooks, M. Karplus, and B. M. Pettitt. Proteins: A Theoretical Perspective of Dynamics, Structure, and Thermodynamics, (John Wiley, New York, 1988).

[3] G. U. Nienhaus, J. R. Mourant, and H. Frauenfelder. Proc. Natl. Acad. Sci. (USA) 89 (1992) 2902.

[4] F. Post, W. Doster, G. Karvounis, and M. Settles. Biophys. J. 64 (1993) 1833. 
[5] F. Parak, E. N. Frolov, A. A. Kononenko, R. L. Mossbauer, and V. I. Goldanskii. FEBS Lett. 117 (1980) 368.

[6] B. F. Rasmussen, A. M. Stock, D. Ringe, and G. A. Petsko. Nature 357 (1992) 423.

[7] M. Ferrand, J. Dianoux, W. Petry, and G. Zaccai. Proc. Natl. Acad. Sci. USA 90 (1993) 9668.

[8] S. G. Cohen, E. R. Bauminger, I. Nowik, S. Ofer, and J. Yariv. Phys. Rev. Lett. 46 (1981) 1244.

[9] F. Parak, E. N. Frolov, R. L. Mossbauer, and V. I. Goldanskii. J. Mol. Biol. 145 (1981) 825.

[10] E. W. Knapp, S. F. Fisher, and F. Parak. J. Am. Chem. Soc. 86 (1982) 5042.

[11] W. Doster, S. Cusack, and W. Petry. Nature 337 (1989) 754.

[12] R. F. Tilton, Jr, J. C. Dewan, and G. A. Petsko. Biochemistry 31 (1992) 2469.

[13] J. L. Green, J. Fan, and C. A. Angell. J. Phys. Chem. 98 (1994) 13780.

[14] J. Fitter, R. E. Lechner, and N. A. Dencher. Biophys. J. 73 (1997) 2126.

[15] V. Réat, R. Dunn, M. Ferrand, J. L. Finney, R. M. Daniel, and J. C. Smith. Proc. Natl. Acad. Sci. USA 97 (2000) 9961.

[16] D. J. Bicout, and G. Zaccai. Biophys. J. 80 (2001) 1115-1123.

[17] A. L. Lee, and A. J. Wang. Nature 411 (2001) 501.

[18] M. M. Teeter, A. Yamano, B. Stec, and U. Mohanty. Proc. Natl. Acad. Sci. USA 98 (2001) 11242.

[19] T. E. Creighton. Curr. Opin. Struct. Biol. 1 (1991) 5.

[20] L. Van Hove. Phys. Rev. 95, 249 (1954).

[21] M. Bée. Quasielastic Neutron Scattering, (Adam Hilger, Bristol, 1988).

[22] D. J. Bicout. Phys. Rev. E 62 (2000) 261.

[23] Smith, J., K. Kuczera, and M. Karplus. Proc. Natl. Acad. Sci. (USA) 87 (1990) 1601.

[24] F. Volino, and A. J. Dianoux. Mol. Phys. 41 (1980) 271.

[25] P. L. Hall, and D. K. Ross. Mol. Phys. 42 (1981) 673.

[26] M.-C. Bellissent-Funel, J. Teixeira, K. F. Bradley, and S. H. Chen. J. Phys. I (France) 2 (1992) 995; M.-C. Bellissent-Funel, J-M. Zanotti and S. H. Chen. Faraday Discuss. 103 (1996) 281 .

[27] Hydration Processes in Biology: Theoretical and Experimental Approaches, Ed. M.-C. BellissentFunel, (IOS press, Amsterdam, 1999, Vol. 305).

[28] Stoeckli et al. Physica B 136 (1986) 161.

[29] J. Fitter, R. E. Lechner, G. Büldt, and N. A. Dencher. Proc. Natl. Acad. Sci. USA 93 (1996) 7600.

[30] J. Perez, J.-M. Zanotti, and D. Durand. Biophys. J. 77 (1999) 454.

[31] In Hydration Processes in Biology: Theoretical and Experimental Approaches, Ed. M.-C. Bellissent-Funel, (IOS press, Amsterdam, 1999, Vol. 305) pp. 195-217.

[32] D. J. Bicout. ILL Proceedings Millennium Symposium (2001) 60-62.

[33] S. Dellerue, A.-J. Petrescu, J. C. Smith, and M.-C. Bellissent-Funel. Biophys. J. 81 (2001) 1666.

[34] V. Réat, H. Patzelt, M. Ferrand, C. Pfister, D. Oesterhelt, and G. Zaccai. Proc. Natl. Acad. Sci. USA 95 (1998) 4970.

[35] L. Cordone, M. Ferrand, E. Vitrano, and G. Zaccai. Biophys. J. 76 (1999) 1043. 